XI International Conference on Computational Plasticity. Fundamentals and Applications

COMPLAS XI

E. Oñate, D.R.J. Owen, D. Peric and B. Suárez (Eds)

\title{
MICROPLANE MODEL M6F FOR FIBER REINFORCED CONCRETE
}

\author{
Ferhun C. Caner* and Zdeněk P. Bažant ${ }^{\dagger}$ \\ * Institute of Energy Technologies (INTE/ETSEIB) \\ Universitat Politecnica de Catalunya \\ Campus Sud, 08028 Barcelona, Spain \\ e-mail:ferhun.caner@upc.edu \\ ${ }^{\dagger}$ Dept. of Civil and Environmental Engineering \\ Northwestern University \\ 2145 Sheridan Rd., Evanston, IL 60208, USA \\ e-mail:z-bazant@northwestern.edu
}

Key words: Microplane model, constitutive modeling, fracture, damage, fiber reinforced, concrete

\begin{abstract}
The new microplane model M6f for fiber reinforced concrete features several improvements over the earlier versions: (i) An explicit volumetric-deviatoric split to no split transition formulation in tension which eliminates spurious contraction under tension that the earlier models suffered; (ii) tension-compression load cycles are now correctly simulated using the loading/unloading rules prescribed in the transition function; (iii) a new micro- macro stress equilibrium equation in which the work of volumetric stresses on deviatoric strains and the work of deviatioric stresses on volumetric strains are explicitly accounted for is introduced to correctly model the pressure sensitive dilatant behavior of low to normal strength concretes; (iv) the volumetric boundary is made a function of the maximum principal strain difference in addition to the volumetric strains, so as to extend the data fitting capability to lower strength concretes; (v) the cohesion in the friction boundary now approaches zero linearly, instead of asymptotically with growing tensile volumetric strains, so as to generate an earlier decaying tail in the uniaxial tension and compression. The material behavior has been verified against various test data from the literature. The fits have been improved compared to the previous versions of the model.
\end{abstract}

\section{INTRODUCTION}

The microplane models, which range from M0 to M6 developed since 1984 primarily for the constitutive behavior of concrete, are hierarchically semi-multiscale models [1], because the angular interactions of inelastic phenomena are captured explicitly whereas 
the interactions at a distance cannot be captured as a result of lumping the inelastic phenomena into a single material point. Consequently, the microplane models have already featured some of the properties of the now fashionable multiscale models. In certain important respect, the hierarchical multiscale models for quasibrittle fracture are no better than the microplane models [1] because both miss the microcrack interactions at a distance and fail to predict the size of the fracture process zone (or the localization limiter), while both can capture the angular interactions.

Microplane models have a number of advantages over the conventional tensorial models. Through the microplane concept, the constitutive model can be specified in terms of vectors instead of stress and strain tensor invariants [3] which removes the dependence on the invariants of stress and strain tensors. The principle of frame indifference is still satisfied, albeit approximately, by virtue of using microplanes that sample without bias all possible orientations in the three-dimensional space. The constitutive laws specified on the microplanes is activated by employing either the kinematic or the static constraints. It is well known that for quasi-brittle materials such as concrete, the softening behavior can only be captured if the kinematic constraint is employed [3]. A selective activation of the constitutive behavior on various microplanes results in a macroscopic constitutive behavior equivalent to multisurface plasticity, one or several surfaces on each microplane. Thus, the advantages of the multisurface plasticity, such as capturing the vertex effect and frictional shear with apparently non-associated dilatancy $[4,5]$, are also exhibited by the microplane models.

The latest of the microplane models for fiber reinforced concrete reported in this paper features several improvements over the earlier version [6]. To remove the spurious lateral contraction under tension, the traditional volumetric-deviatoric split formulation [4] gradually becomes the formulation without a split [3], in a form proposed by G. di Luzio as a function of increasing tensile strains. Thus, under tension, the new model predominantly behaves as a model without volumetric-deviatoric split. Also, with the new formulation, the model correctly features extensional damage as observed in the loading-unloading tensile-compressive tests reported in the literature.

Furthermore, the experimental data reported on specimens of low strength concretes [7], which could not be fitted well with the earlier versions of the model, can now be fitted well. To this end, the volumetric-deviatoric coupling is extended to the volumetric boundary. To account for the cross coupling of shear and dilatancy more accurately, the macro-micro stress equilibrium equation is also modified: The normal microplane stress is now treated directly as the sum of the volumetric and deviatoric parts without any additional constraints. Thus, the requirements of vanishing work of the deviatoric stresses on the volumetric strains, and of the volumetric stresses on the deviatoric strains, which was postulated in models M4, M5 and M5f, is now removed [4, 9, 10]. As a result, the predictive capabilities of the model have been drastically improved. 


\section{BASIC CONSTITUTIVE EQUATIONS OF MICROPLANE MODEL}

Microplane models for concrete are defined using the kinematic constraint, which means that microplane strains are projections of the strain tensor on the microplanes $[3,4,9,10]$ :

$$
\epsilon_{N}=n_{i} n_{j} \epsilon_{i j}=N_{i j} \epsilon_{i j}
$$

where $n_{i}$ are the components of the microplane normal vectors and $i, j=1,2,3$ are the indices of the cartesian coordinate system. Alternatively, a static constraint in which the stress tensor is projected on the microplanes to yield the microplane stresses is also possible, but this approach is useful mainly for modeling hardening type inelastic behavior in which no softening can take place. For the modeling of softening, it is essential to use the kinematic constraint as given by Eq.1 [?, 3].

The projection of the strain tensor on the microplane system results in microplane shear strain vectors. However, to be able to fit some of the unconventional experimental data on concrete, it was found essential that the microplane shear strain vectors be represented with respect to in-plane orthogonal directions given by the randomly generated in-plane microplane vectors $\vec{m}$ and $\vec{l}$. Thus, the shear strains on the microplanes are defined as

$$
\epsilon_{M}=\frac{1}{2}\left(n_{i} m_{j}+n_{j} m_{i}\right) \epsilon_{i j}=M_{i j} \epsilon_{i j} ; \epsilon_{L}=\frac{1}{2}\left(n_{i} l_{j}+n_{j} l_{i}\right) \epsilon_{i j}=L_{i j} \epsilon_{i j}
$$

To separate the normal response into its volumetric and deviatoric parts, which is necessary for being able to model linear elasticity by means of the microplane model [4], we define the relations

$$
\epsilon_{N}=\epsilon_{V}+\epsilon_{D} ; \sigma_{N}=\sigma_{V}+\sigma_{D}
$$

where $\epsilon_{V}=\epsilon_{k k} / 3$ and $\epsilon_{D}=\left(N_{i j}-\delta_{i j} / 3\right) \epsilon_{i j}$. The microplane volumetric and deviatoric stresses $\sigma_{V}$ and $\sigma_{D}$ as well as the microplane shear strains $\sigma_{M}$ and $\sigma_{L}$ must be prescribed as functions of the microplane strains. These relations are the microplane constitutive laws which must be determined through data fitting:

$$
\sigma_{V}=\mathcal{F}_{V}\left(\epsilon_{V}, \sigma_{I}, \sigma_{I I I}\right) ; \sigma_{D}=\mathcal{F}_{D}\left(\epsilon_{D}, \epsilon_{V}\right) ; \sigma_{L}=\mathcal{F}_{T}\left(\epsilon_{L}, \epsilon_{V}, \sigma_{N}\right) ; \sigma_{M}=\mathcal{F}_{T}\left(\epsilon_{M}, \epsilon_{V}, \sigma_{N}\right)
$$

Although the constitutive relation for the microplane shear strain components $\sigma_{L}$ and $\sigma_{M}$ are given by the same shear law in Eq.4, this is actually not strictly necessary; one may assume an in-plane orthotropy of microplanes and prescribe different shear laws for the two orthogonal shear components on the microplane as well.

When the microplane constitutive laws depend on some measure of stress, they become implicit. For the modeling of highly inelastic frictional behavior, it is desirable to achieve explicit microplane constitutive laws which would be free of iterations. Explicit constitutive laws are always sought in the development of microplane models. However, in some constitutive laws, it was inevitable to introduce stresses as the independent variables. For example, the shear behavior must involve the normal stresses because in the 
inelastic range it is the friction that is modeled, which is by definition a function of the normal stresses. For volumetric behavior, it is simpler to define the deviatoric effects in terms of the principal stress difference.

\subsection{Normal Boundaries}

The normal boundary governs the tensile fracturing behavior of the model. It is expressed as:

$$
\sigma_{N}^{b}=F_{N}\left(\epsilon_{N}\right)=E k_{1} c_{1} \exp \left(-\frac{\left\langle\epsilon_{N}-c_{1} c_{2} k_{1}\right\rangle}{k_{1} c_{3}+c_{4}\left\langle-\sigma_{V} / E_{V}\right\rangle}\right)
$$

\subsection{Deviatoric Boundaries}

The deviatoric boundaries simulate the spreading and splitting cracks under compression. They are given by:

$$
\begin{gathered}
\sigma_{D}^{b+}=F_{D}^{+}\left(\epsilon_{D}\right)=\frac{E k_{1} \beta_{1}}{1+\left(\left\langle\epsilon_{D}-\beta_{1} \beta_{2} k_{1}\right\rangle /\left(k_{1} c_{17} \beta_{3}\right)\right)^{2}} \\
\sigma_{D}^{b-}=F_{D}^{-}\left(-\epsilon_{D}\right)=\frac{E k_{1} \beta_{4}}{1+\left(\left\langle-\epsilon_{D}-\beta_{4} \beta_{5} k_{1}\right\rangle /\left(k_{1} \beta_{3}\right)\right)^{2}}
\end{gathered}
$$

where

$$
\beta_{1}=c_{18} \exp \left(-\left(f_{c}^{\prime} / E-f_{c 0}^{\prime} / E_{0}\right)\right) \tanh \left(c_{19}\left\langle-\epsilon_{V}\right\rangle / k_{1}\right)\left(1+\chi_{1}\right)+\chi_{1}+c_{5}
$$

where $\chi_{1}=k_{9} \tanh \left(c_{29} V_{f}\right)$;

$$
\begin{gathered}
\beta_{2}=c_{6} \exp \left(-\left(f_{c}^{\prime} / E-f_{c 0}^{\prime} / E_{0}\right)\right) \min \left[\exp \left(c_{20}\left\langle-\epsilon_{V} / k_{1}\right\rangle\right), c_{21}\right] \\
\beta_{3}=c_{22} \exp \left(-\left(f_{c}^{\prime} / E-f_{c 0}^{\prime} / E_{0}\right)\right) \tanh \left(c_{19}\left\langle-\epsilon_{V}\right\rangle / k_{1}\right)\left(1+\chi_{2}\right)+\chi_{2}+c_{7}
\end{gathered}
$$

where $\chi_{2}=k_{10} \tanh \left(c_{29} V_{f}\right)$;

$$
\begin{gathered}
\beta_{4}=c_{23} \exp \left(-\left(f_{c}^{\prime} / E-f_{c 0}^{\prime} / E_{0}\right)\right) \tanh \left(c_{19}\left\langle-\epsilon_{V}\right\rangle / k_{1}\right)\left(1+\chi_{1}\right)+\chi_{1}+c_{8} \\
\beta_{5}=c_{9} \exp \left(-\left(f_{c}^{\prime} / E-f_{c 0}^{\prime} / E_{0}\right)\right) \min \left[\exp \left(c_{20}\left\langle-\epsilon_{V} / k_{1}\right\rangle\right), c_{21}\right]
\end{gathered}
$$

The functions $\chi_{1}, \chi_{2}$ represent the contribution of the fibers to resist the compressive splitting and slip cracks. The compressive strength may be calculated using the ACI formula in $\mathrm{MPa} f_{c}^{\prime}=(E / 5150.226)^{2}$ if it is not given.

\subsection{Frictional Yield Surface}

The frictional yield surface simulates the shear behavior of the model. It is given by:

$$
\sigma_{T}^{b}=F_{T}\left(-\sigma_{N}\right)=\frac{E_{T} k_{1} k_{2} c_{10}\left\langle-\sigma_{N}+\sigma_{N}^{0}\right\rangle}{E_{T} k_{1} k_{2}+c_{10}\left\langle-\sigma_{N}+\sigma_{N}^{0}\right\rangle}
$$


where

$$
\sigma_{N}^{0}=\left\langle E_{T} k_{1} c_{11}-c_{12}\left\langle\epsilon_{V}\right\rangle\right\rangle
$$

Under compression, the best data fits are obtained when the shear boundary is applied to the microplane shear components before the peak load is reached, especially in fitting the strength envelopes. In model M6f, a formulation that transits from application of the shear boundary to the microplane shear components to the microplane shear resultant is introduced. The formulation involves calculating the in-plane microplane shear components twice, once by applying the shear boundary to the microplane shear components, and once by applying it to the microplane shear resultant. Then, the transition formulation can be expressed as:

$$
\left.\left.\sigma_{L}=\hat{\sigma_{L}} \tilde{\phi}+\overline{\sigma_{L}}(1-\tilde{\phi})\right) ; \sigma_{M}=\hat{\sigma_{M}} \tilde{\phi}+\sigma_{M}(1-\tilde{\phi})\right)
$$

where the quantities with ^ are those obtained by applying the shear boundary to the microplane shear components, and the quantities with ${ }^{-}$are those obtained by applying the shear boundary to the microplane shear resultant; the transition parameter is defined as:

$$
\tilde{\phi}=\exp \left(c_{27}\left\langle\epsilon_{V}-c_{28}\right\rangle\right)
$$

\subsection{Volumetric Boundary}

The volumetric boundary simulates the pore collapse and expansive breakup of the material. It is given by :

$$
\sigma_{V}^{b}=F_{V}^{-}\left(-\epsilon_{V}\right)=-E k_{1} k_{3} \exp \left(\frac{-\epsilon_{V}}{k_{1} \beta_{6}}\right)
$$

where

$$
\beta_{6}=\frac{k_{5}\left(\sigma_{I}-\sigma_{I I I}\right)}{k_{1}\left(1+\left\langle-\sigma_{V} / E_{V}\right\rangle\right)}+k_{4}
$$

and $\sigma_{I}=$ maximum principal stress; $\sigma_{I I I}=$ minimum principal stress.

\subsection{Unloading and Stiffness Degradation}

The unloading behaviors of concrete under tension and compression are radically different. Under tension the unloading slope is close to the secant modulus, and under compression, on the average it is close to the elastic slope. For $\sigma_{I}>c_{26}$, where $c_{26}$ is a tensile stress threshold, the tension-tension and the tension-compression load cycles can be successfully obtained using the loading-unloading rules prescribed in terms of the 
transition function $\phi$ as follows:

$$
\phi\left(\epsilon_{N}\right)= \begin{cases}\exp \left(-c_{24} \sqrt{\left\langle\epsilon_{N} / k_{1}-c_{25}+\min \left(\epsilon_{I}^{u n l}, c_{25}\right)\right\rangle}\right) & \text { if } \epsilon_{N} / k_{1}<\epsilon_{I}^{u n l}-\min \left(\epsilon_{I}^{u n l}, c_{25}\right) \\ \exp \left(-c_{24} \sqrt{\left\langle\epsilon_{I}^{u n l}-c_{25}\right\rangle}\right) & \text { if } \epsilon_{I}^{u n l}-\min \left(\epsilon_{I}^{u n l}, c_{25}\right) \leq \epsilon_{N} / k_{1}<\epsilon_{I}^{u n l} \\ \exp \left(-c_{24} \sqrt{\left\langle\epsilon_{N} / k_{1}-c_{25}\right\rangle}\right) & \text { if } \epsilon_{N} / k_{1} \geq \epsilon_{I}^{u n l}\end{cases}
$$

where $\epsilon_{I}^{u n l}$ is the value of the maximum principal strain at the initiation of unloading and it is a history parameter. When $\sigma_{I} \leq c_{26}$, there is no tension and thus $\phi=1$.

The loading with a transition from split to no split, along with the unloading, are depicted in Fig. 1(a). For the initial virgin loading, the load path with a transition to no split formulation is the path [ABDC]. At any given point where the unloading starts, for example at point $\mathrm{D}$, the unloading path is given by [DEFA]. This results into a transition from the no split formulation into the split formulation. The subsequent loading passing into the tension zone follows the path [AFEDC]. The length $|D E|=c_{25}$.

As in model M4, the unloading under triaxial compression at high pressures is governed by the unloading rule of the volumetric boundary given by $C_{V}^{u}\left(-\epsilon_{V},-\sigma_{V}\right)=$

$E_{V}\left(c_{13} /\left(c_{13}-\epsilon_{V}\right)+\sigma_{V} /\left(c_{13} c_{14} E_{V}\right)\right)$. As in model M4, the unloading under compression at low confining pressures is governed by the unloading rules of the deviatoric and frictional boundaries. The unloading rule for the compressive deviatoric boundary is givenby $C_{-D}^{u}=$ $\left(1-c_{15}\right) E_{D}+c_{15} E_{D}^{s}$ where $E_{D}^{s}=\min \left(\sigma_{D} / \epsilon_{D}, E_{D}\right)$ for $\sigma_{D} \epsilon_{D}>0$ and $E_{D}^{s}=E_{D}$ for $\sigma_{D} \epsilon_{D} \leq 0$. The unloading rule for the tensile deviatoric boundary is given by $C_{+D}^{u}=$ $\left(1-c_{16}\right) E_{D}+c_{16} E_{D}^{s}$.

The unloading rule of the friction boundary is that the unloading slope is the same as the deviatoric unloading slope; thus

$$
C_{T}^{u}= \begin{cases}C_{-D}^{u} & \text { if } C_{D}^{u}=C_{-D}^{u} \\ C_{+D}^{u} & \text { if } C_{D}^{u}=C_{+D}^{u}\end{cases}
$$

\subsection{Fiber Constitutive Relation}

When the cracks are of the opening mode, the contribution of fiber to the crack bridging stress is given by a simplified form of Kholmyansky's equation [11]:

$$
\frac{\sigma_{N}^{f}}{E}=\left\{\begin{array}{lc}
k_{6} k_{1}\left\langle\epsilon_{N} / k_{1}\right\rangle \exp \left(-k_{7}\left\langle\epsilon_{N} / k_{1}\right\rangle\right) & \text { if } \epsilon_{N} / k_{1}<1 / k_{7} \\
k_{6} k_{1} / k_{7} \exp (-1) & \text { if } 1 / k_{7} \leq \epsilon_{N} / k_{1}<k_{8} \\
k_{6} k_{1}\left\langle\epsilon_{N} / k_{1}-k_{8}+1 / k_{7}\right\rangle \exp \left(-k_{7}\left\langle\epsilon_{N} / k_{1}-k_{8}+1 / k_{7}\right\rangle\right) & \text { if } k_{8} \leq \epsilon_{N} / k_{1}
\end{array}\right.
$$

and the fiber and the matrix are assumed to be coupled in parallel. Thus the result is

$$
\sigma_{N}^{b f}=\sigma_{N}^{b}+\sigma_{N}^{f}
$$

where $\sigma_{N}^{b f}=$ total normal boundary for fiber reinforced concrete, $\sigma_{N}^{b}=$ boundary for plain concrete matrix and $\sigma_{N}^{f}=$ contribution of the fiber given by Eq.21. 


\subsection{Thermodynamic Dissipation}

The thermodynamic dissipation can be formulated as the energy dissipated by deviatoric, volumetric and shear stresses in the case of formulation with a deviatoric-volumetric split. In the case of the formulation without a split, it can be formulated as the energy dissipated by the normal and shear stresses. Under isothermal conditions, the energy dissipated by a microplane stress is given by

$$
T s_{i n t}=\int_{0}^{\epsilon} \sigma d \epsilon-\frac{\sigma^{2}}{2 C^{u}}
$$

where $E_{s} \leq C^{u} \leq E$ with $E_{s}=$ secant modulus. When the loading switches to unloading before reaching any of the boundaries, the response is elastic, and thus such the load cycles do not increase dissipation. When the boundaries are reached during loading, energy dissipation takes place as given by Eq.(23). The unloading slope is assumed to be constant although it has a slight curvature for the volumetric boundary. Thus, the energy dissipated by the deviatoric, volumetric, normal and shear stresses may be expressed as

$$
\begin{aligned}
& T s_{i n t}^{D}=\int_{0}^{\epsilon_{D}} \sigma_{D} d \epsilon_{D}+\int_{0}^{\epsilon_{v}} \sigma_{D} d \epsilon_{V}-\frac{\sigma_{D}^{2}}{2 C_{D}^{u}} ; T s_{i n t}^{V}=\int_{0}^{\epsilon_{D}} \sigma_{V} d \epsilon_{D}+\int_{0}^{\epsilon_{v}} \sigma_{V} d \epsilon_{V}-\frac{\sigma_{V}^{2}}{2 C_{V}^{u}} \\
& T s_{i n t}^{N}=\int_{0}^{\epsilon_{N}} \sigma_{N}^{*} d \epsilon_{N}-\frac{\left(\sigma_{N}^{*}\right)^{2}}{2 C_{N}^{u}} ; T s_{i n t}^{L}=\int_{0}^{\epsilon_{L}} \sigma_{L} d \epsilon_{L}-\frac{\sigma_{L}^{2}}{2 C_{T}^{u}} ; T s_{i n t}^{M}=\int_{0}^{\epsilon_{M}} \sigma_{M} d \epsilon_{M}-\frac{\sigma_{M}^{2}}{2 C_{T}^{u}}
\end{aligned}
$$

Consequently, the total energy dissipation per unit volume of material is, in model M6f, approximately given by

$$
T \tilde{s}_{i n t}=T \frac{3}{2 \pi} \int_{\Omega}\left[\left(s_{i n t}^{D}+s_{i n t}^{V}\right) \phi+s_{i n t}^{N}(1-\phi)+s_{i n t}^{L}+s_{i n t}^{M}\right] d \Omega
$$

\section{MICRO-MACRO STRESS EQUILIBRIUM}

\subsection{The model with volumetric-deviatoric split}

The microplane model with volumetric-deviatoric split in the new microplane model M6 is defined as in the model M2:

$$
\begin{aligned}
\frac{2 \pi}{3} \sigma_{i j} \delta \epsilon_{i j} & =\int_{\Omega}\left[\sigma_{D} \delta \epsilon_{D}+\sigma_{V} \delta \epsilon_{V}+\sigma_{M} \delta \epsilon_{M}+\sigma_{L} \delta \epsilon_{L}\right] d \Omega+\int_{\Omega}\left[\sigma_{D} \delta \epsilon_{V}+\sigma_{V} \delta \epsilon_{D}\right] d \Omega \\
\frac{2 \pi}{3} \sigma_{i j} \delta \epsilon_{i j} & =\int_{\Omega}\left[\left(\sigma_{D}+\sigma_{V}\right)\left(\delta \epsilon_{N}-\delta \epsilon_{V}\right)+\left(\sigma_{V}+\sigma_{D}\right) \delta \epsilon_{V}+\sigma_{M} \delta \epsilon_{M}+\sigma_{L} \delta \epsilon_{L}\right] d \Omega \\
\frac{2 \pi}{3} \sigma_{i j} \delta \epsilon_{i j} & =\int_{\Omega}\left[\sigma_{N} \delta \epsilon_{N}+\sigma_{M} \delta \epsilon_{M}+\sigma_{L} \delta \epsilon_{L}\right] d \Omega \\
\Rightarrow \sigma_{i j} & =\frac{3}{2 \pi} \int_{\Omega}\left[\sigma_{N} N_{i j}+\sigma_{M} M_{i j}+\sigma_{L} L_{i j}\right] d \Omega
\end{aligned}
$$


Ferhun C. Caner and Zdeněk P. Bažant

where $\sigma_{N}=\sigma_{V}+\sigma_{D}$. The last integral on the right hand side of Eq.(25) did not exist in the model M4, because it was postulated that the work of deviatoric stresses on the volumetric strains and the work of volumetric stresses on the deviatoric strains did not exist. Thus the volumetric stresses were work-conjugate to only the volumetric strains and the deviatoric stresses only to the deviatoric strains.

However, the triaxial compressive test data for low strength concretes, for which the behavior is more dissipative and ductile than the normal strength concretes, could not be fitted using the model M5f. It was found out that, these test data could be fitted easily if an explicit volumetric-deviatoric coupling could be assumed. Thus, the macro-micro stress equilibrium formula employed in model M5f has been replaced with that employed in M2 and M3, given by Eq.28. In general, it was found out that, as the strength of concrete increases, such a coupling must have lesser effect because the material becomes more brittle, with a progressively shrinking cohesive zone. For very high strength concretes, the size of the cohesive zone essentially becomes sub-millimeter, and the crack surfaces become smoother. This means that the frictional nature of the material becomes much less significant as the strength of the concrete increases compared to the normal strength concrete.

\subsection{The model without volumetric-deviatoric split}

The macro-micro stress equilibrium equation for the kinematically constrained microplane model without volumetric-deviatoric split can be written as $\frac{2 \pi}{3} \sigma_{i j}^{*} \delta \epsilon_{i j}^{*}=$ $\int_{\Omega}\left[\sigma_{N}^{*} \delta \epsilon_{N}^{*}+\sigma_{M}^{*} \delta \epsilon_{M}^{*}+\sigma_{L}^{*} \delta \epsilon_{L}^{*}\right] d \Omega$, leading to

$$
\Rightarrow \sigma_{i j}^{*}=\frac{3}{2 \pi} \int_{\Omega}\left[\sigma_{N}^{*} N_{i j}+\sigma_{M}^{*} M_{i j}+\sigma_{L}^{*} L_{i j}\right] d \Omega
$$

where $\epsilon_{N}^{*}=N_{i j} \epsilon_{i j}^{*}, \epsilon_{M}^{*}=M_{i j} \epsilon_{i j}^{*}, \epsilon_{L}^{*}=L_{i j} \epsilon_{i j}^{*} ; \sigma_{N}^{*}=\mathcal{F}_{N}^{*}\left(\epsilon_{N}^{*}\right), \sigma_{M}=\mathcal{F}_{T}^{*}\left(\epsilon_{M}^{*}, \sigma_{N}^{*}\right)$ and $\sigma_{L}^{*}=\mathcal{F}_{T}^{*}\left(\epsilon_{L}^{*}, \sigma_{N}^{*}\right)$. In this model, the microplane normal stress is directly calculated as a function of the microplane normal strains.

\subsection{The model with transition}

The present model, model M6f, employs an explicit transition formulation, in which the model with a split given by Eq.28 gradually transits to the model without a split given by Eq.29;

$$
\sigma_{i j}=\frac{3}{2 \pi} \int_{\Omega}\left[\left\{\sigma_{N}^{*}(1-\phi)+\sigma_{N} \phi\right\} N_{i j}+\sigma_{M} M_{i j}+\sigma_{L} L_{i j}\right] d \Omega
$$

where $\sigma_{M}=\sigma_{M}^{*}$ and $\sigma_{L}=\sigma_{L}^{*}$. The transition function $\phi$ for monotonic loading is defined as

$$
\phi\left(\epsilon_{N}\right)=\left\{\begin{array}{cl}
\exp \left(-c_{24} \sqrt{\left\langle\epsilon_{I} / k_{1}-c_{25}\right\rangle}\right) & \text { if } \sigma_{I}>c_{26} \\
1 & \text { if } \sigma_{I} \leq c_{26}
\end{array}\right.
$$

and thus is active only under tension. 
Ferhun C. Caner and Zdeněk P. Bažant

\section{RESULTS AND DISCUSSION}

The simulations to predict experimental data for Harex and PVA fiber reinforced concretes under uniaxial tension are shown in Fig.1(b),(c) [8]; and those for steel fiber reinforced concretes under uniaxial compression and triaxial compression are shown in Figs.1(d)-(f) [7] respectively. The agreement between the data and the predictions is quite satisfactory. The model parameters, their values and their brief descriptions, are shown in Table 1. The "c" parameters are fixed parameters which do not change from one concrete to another; the " $\mathrm{k}$ " parameters may change from one concrete to another and thus they must be calibrated for each given concrete. Although there seem to be too many parameters, only 5 of them are free parameters for the concrete matrix and another 5 free parameters are for the fiber effect on the opening, splitting and slipping type fracture which must be supplied by the user of the model. For most low to normal strength concretes, the values provided in Table 1 should be sufficient. For others, identification of these free parameters $\left(k_{1}\right.$ to $\left.k_{5}\right)$ using test data conducted on specimens of the concrete under consideration may be necessary. For various types of fibers, the values of free fiber parameters $\left(k_{6}\right.$ to $\left.k_{10}\right)$ are already determined as shown in Table 1 . If a new type of fiber used, these values should be recalibrated by fitting uniaxial tension and uniaxial compression test data.

Table 1: Parameters of the model M6f, their typical values and their meanings.

\begin{tabular}{rcl}
\hline \hline par. & value & meaning \\
\hline$f_{c 0}^{\prime}$ & $15.08 \mathrm{MPa}$ & reference compressive strength \\
\hline$E_{c 0}$ & $20 \mathrm{GPa}$ & reference elastic modulus \\
\hline$c_{1}$ & 0.46 & controls the uniaxial tensile strength \\
\hline$c_{2}$ & 2.76 & controls the roundness of the peak in uniaxial tension \\
\hline$c_{3}$ & 4 & controls the slope of the postpeak in uniaxial tension \\
\hline$c_{4}$ & 70 & controls the slope of the postpeak tail in uniaxial compression \\
\hline$c_{5}$ & 2.5 & controls the vol. expansion under compression \\
\hline$c_{6}$ & 1.3 & controls the roundness of the peak in vol. expansion under compression \\
\hline$c_{7}$ & 50 & controls the slope of the initial postpeak in uniaxial compression \\
\hline$c_{8}$ & 8 & controls the peak strength in uniaxial compression \\
\hline$c_{9}$ & 1.3 & controls the peak roundness in uniaxial compression \\
\hline$c_{10}$ & 0.73 & controls the effective friction coefficient \\
\hline$c_{11}$ & 0.2 & initial cohesion in frictional response \\
\hline$c_{12}$ & $7 \cdot 10^{3}$ & controls the change of cohesion with tensile vol. strains \\
\hline$c_{13}$ & 0.02 & controls the unloading slope at high hydrostatic compression \\
\hline$c_{14}$ & 0.01 & controls the unloading slope at low hydrostatic compression \\
\hline$c_{15}$ & 0.4 & controls the unloading slope of the compressive dev. boundary \\
\hline$c_{16}$ & 0.99 & controls the unloading slope of the tensile dev. boundary \\
\hline$c_{17}$ & 0.082 & controls the tensile cracking under compression \\
\hline
\end{tabular}


Ferhun C. Caner and Zdeněk P. Bažant

Table 1 - continued from previous page

\begin{tabular}{ccl}
\hline par. & value & meaning \\
\hline$c_{18}$ & 4 & controls the vol. expansion for low strength concretes \\
\hline$c_{19}$ & 0.012 & controls the vol. expansion rate for low strength concretes \\
\hline$c_{20}$ & 400 & controls the roundness of vol. expansion for low strength concretes \\
\hline$c_{21}$ & 13 & controls the roundness of vol. expansion for low strength concretes \\
\hline$c_{22}$ & $3.5 \cdot 10^{3}$ & controls the roundness of vol. expansion for low strength concretes \\
\hline$c_{23}$ & 20 & controls the roundness of vol. expansion for low strength concretes \\
\hline$c_{24}$ & 0.12845 & controls the rate of transition from split to no split \\
\hline$c_{25}$ & 0.7576 & controls unloading under tension \\
\hline$c_{26}$ & $1.7 \cdot 10^{-4}$ & tensile stress treshold for initiation of split to no split transition \\
\hline$c_{27}$ & $1 \cdot 10^{3}$ & rate of transition from shear boundary over shear components to over resultant \\
\hline$c_{28}$ & $5 \cdot 10^{-4}$ & threshold of vol. strain to start the above transition \\
\hline$c_{29}$ & 100 & fiber contribution to resist splitting and slipping \\
\hline$k_{1}$ & $1.5 \cdot 10^{-4}$ & radial scaling parameter \\
\hline$k_{2}$ & 500 & controls the horizontal asymptote value in the frictional boundary \\
\hline$k_{3}$ & 15 & controls the shape of the volumetric boundary \\
\hline$k_{4}$ & 150 & controls the shape of the volumetric boundary \\
\hline$k_{5}$ & 2 & controls the triaxial hardening for low strength concretes at low pressures \\
\hline$k_{6}$ & 0.357 & fiber law vertical scaling \\
\hline$k_{7}$ & 0.2345 & fiber law softening rate \\
\hline$k_{8}$ & 5.09 & controls the length of fiber law horizontal plateau \\
\hline$k_{9}$ & 0.25 & fiber contribution to resist splitting and slipping \\
\hline$k_{10}$ & 240 & fiber contribution to resist splitting and slipping \\
\hline$V_{f}$ & 0.02 & fiber volume fraction \\
\hline & &
\end{tabular}

\section{CONCLUSIONS}

A new, improved microplane model, called M6f, for mechanical behavior of fiber reinforced concretes has been reported. The new model improves upon its predecessors (i) by eliminating the spurious contraction under tension that the earlier models suffered, (ii) by predicting the tension-compression load cycles correctly, unlike the earlier versions, (iii) by extending range of experimental data fitted to lower strength concretes. The model performance has been calibrated and verified against numerous test data from the literature.

Acknowledgment: The work of the second author was supported by Grant W911NF09-1-0043 from ARO Durham, monitored by James O'Daniel, Kent Danielson and Marc Adley of ERDC, Vicksburg, and partly also by the U.S. DoT through NU Infrastructure Technology Institute Grant 60027740. 
Ferhun C. Caner and Zdeněk P. Bažant

\section{REFERENCES}

[1] Bažant, Z.P. Can Multiscale-Multiphysics Methods Predict Softening Damage and Structural Failure?, Mechanics of the American Academy of Mechanics (AAM) (2007) 36(5-6):5-12.

[2] Bažant, Z.P. Microplane model for strain-controlled inelastic behavior. Chapter 3 in "Mechanics of Engineering Materials." ed. by C. S. Desai and R. H. Gallagher, J. Wiley, London (1984) 45-59.

[3] Bažant, Z. P., and Oh, B.-H. Microplane model for progressive fracture of concrete and rock. ASCE J. of Engrg. Mechanics (1985) 111:559-582.

[4] Bažant, Z. P., Caner, F. C., Carol, I., Adley, M. D., and Akers, S. A. Microplane model M4 for concrete: I. Formulation with work-conjugate deviatoric stress. ASCE J. Eng. Mech. (2000) 126(9): 944-953.

[5] Caner, F. C., Bažant, Z. P., and Červenka, J. Vertex effect in strain-softening concrete at rotating principal axes. ASCE J. Eng. Mech. (2002) 128(1): 24-33.

[6] Beghini, A., Bažant, Z.P., Zhou, Y., Gouirand, O. and Caner, F. C. Microplane Model M5f for Multiaxial Behavior and Fracture of Fiber-Reinforced Concrete. ASCE J. of Eng. Mech. (2007) 133(1):66-75

[7] Chern, J.-C., Yang, H.-J., and Chen, H.-W. Behavior of steel fiber-reinforced concrete in multiaxial loading. ACI Mater. J. (1992) 891:32-40.

[8] Li, F., and Li, Z. Continuum damage mechanics based modeling of fiber-reinforced concrete in tension. Int. J. Solids Struct. (2001) 38:777-793.

[9] Bažant, Z. P., and Caner, F. C. Microplane model M5 with kinematic and static constraints for concrete fracture and anelasticity. I. Theory. J. Eng. Mech. (2005) $131(1): 31-40$.

[10] Bažant, Z. P., and Caner, F. C. Microplane model M5 with kinematic and static constraints for concrete fracture and anelasticity. II. Computation. J. Eng. Mech. (2005) 131(1):41-47.

[11] Kholmyansky, M. M. Mechanical resistance of steel fiber reinforced concrete to axial load. J. Mater. Civ. Eng. (2002)144:311-319. 


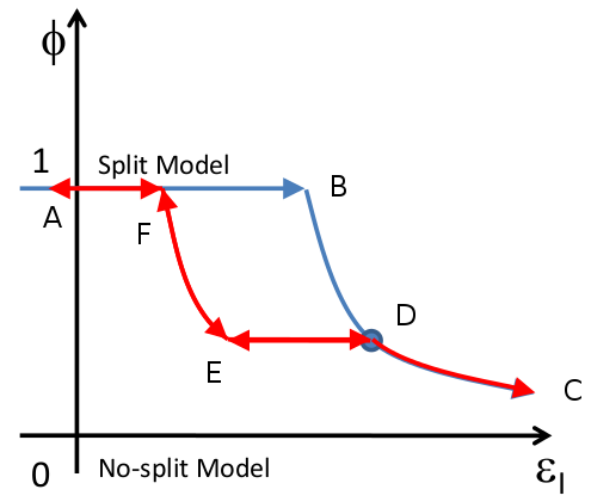

(a)

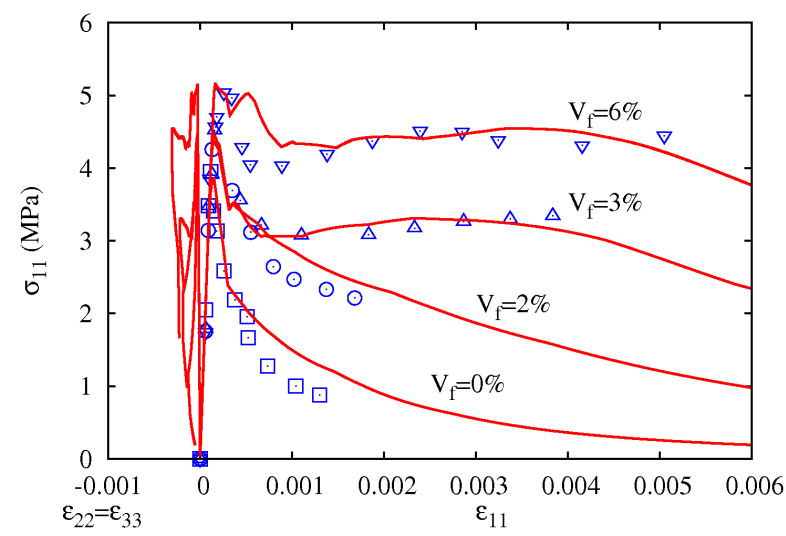

(c)

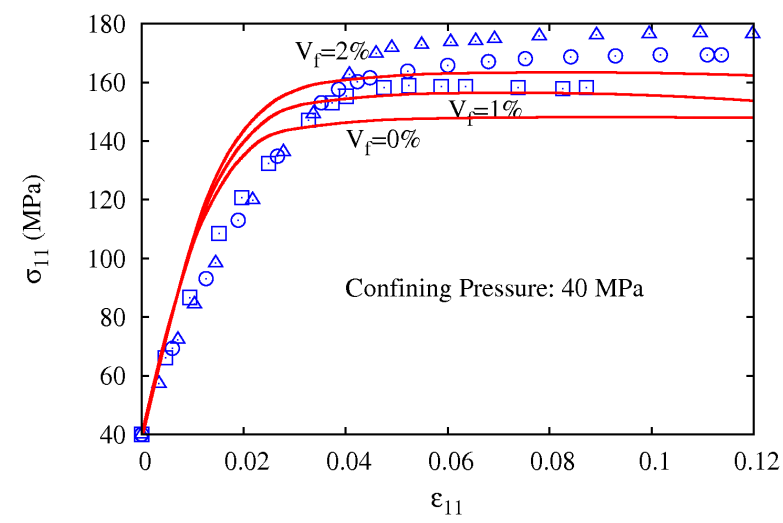

(e)

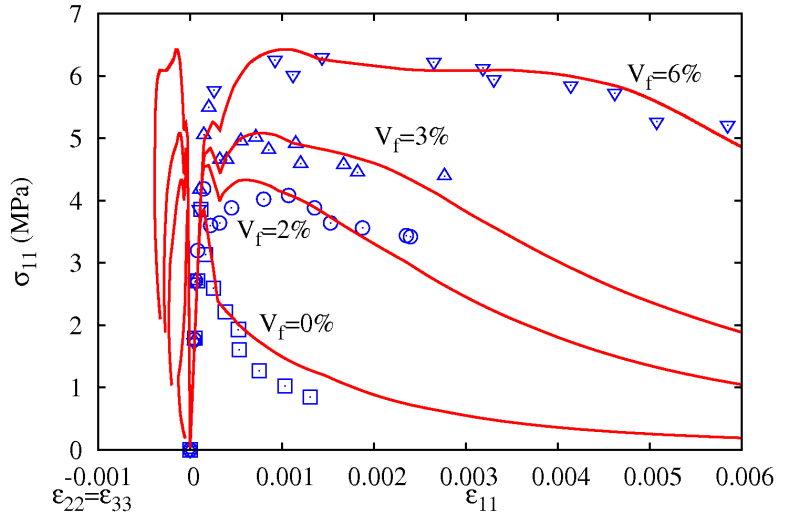

(b)

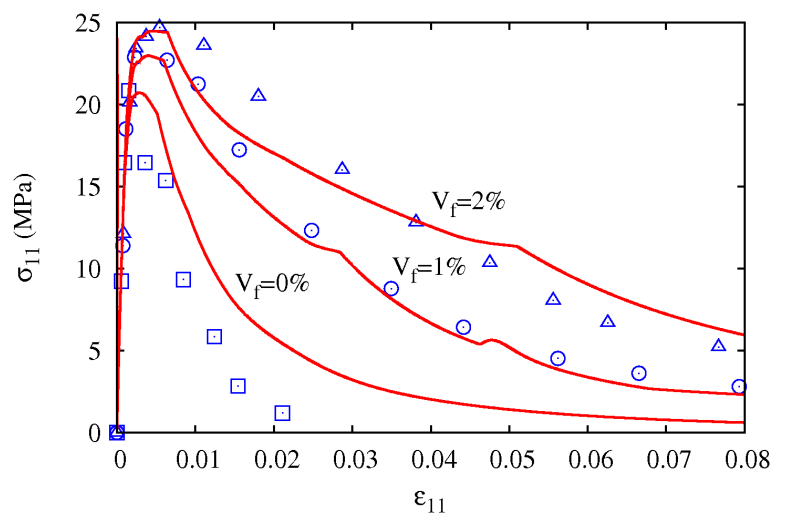

(d)

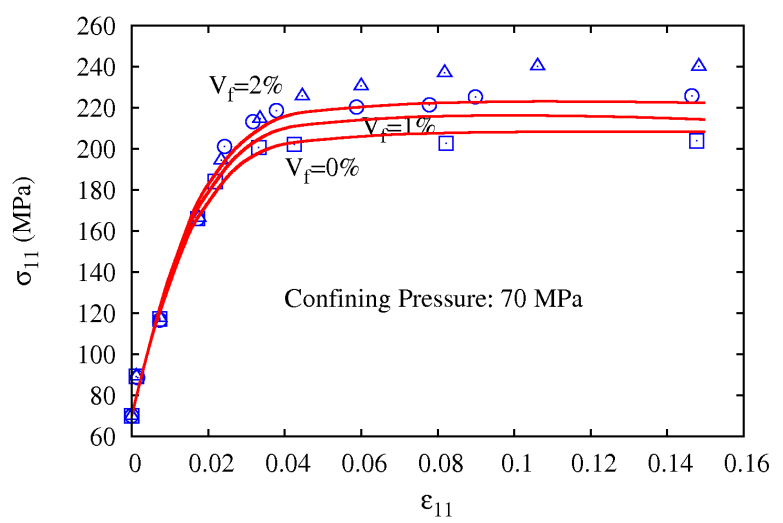

(f)

Figure 1: (a) the loading/unloading rule under tension; test data with its prediction by the model M6f for (b) Harex [8], (c) PVA [8], (d),(e) and (f) carbon steel fiber [7] reinforced concrete. 